\title{
Mecânica Clássica - Trajetórias e o conceito de tempo
}

\author{
Mario Cezar Bertin ${ }^{1}$ \\ ${ }^{1}$ Instituto de Física, Universidade Federal da Bahia
}

September 24, 2020

Rich media available at https ://youtu.be/cy9LSFUaRbE

\section{O postulado 3}

Até este ponto de nossa tentativa de axiomatização da mecânica clássica, introduzimos dois postulados:

1. Postulado 1: A posição de uma partícula é representada por um ponto em $\mathbb{R}^{3}$.

2. Postulado 2: A distância entre duas partículas é definida pela métrica euclidiana.

Com estes pontulados, discutimos os conceitos de

1. Partícula e Interação;

2. O espaço euclidiano e suas simetrias;

3. Observáveis euclidianos,

como integrantes fundamentais da teoria.

Agora, devemos introduzir outros dois postulados. O primeiro sedimenta a ideia de como o movimento das partículas é representado no espaço euclidiano e se suporta na percepção de que uma partícula não pode ser criada ou destruída. Neste caso, se uma partícula se move de um ponto $A$ a um ponto $B$, ele deve percorrer um conjunto contínuo de pontos entre esses pontos, passando por todos os pontos. Este conjunto de pontos é denominado trajetória. Por enquanto, é irrelevante a razão do movimento.

Postulado 3: O movimento de uma partícula é representado por uma curva suave no espaço euclidiano tridimensional.

Assim, apresentamos formalmente o conceito de movimento e seu correspondente do lado matemático do mapeamento de que tanto discutimos; a curva.

\section{Curvas e Trajetórias em $\mathbb{R}^{3}$}

Uma curva é uma relação entre um intervalo fechado da reta real e o espaço euclidiano:

$$
\gamma: I=\left[t_{0}, t\right] \subset \mathbb{R} \rightarrow \mathbb{R}^{3} .
$$

Portanto, um subconjunto de números reais, que constitui o domínio da curva, é relacionado a um conjunto de pontos, o conjunto imagem de $\gamma$, do espaço euclidiano, que constutui seu contra-domínio. 
Aqui, vamos diferenciar o conceito de curva e de trajetória. Uma trajetória é composta pelo conjunto imagem da curva e é denotada por um conjunto de equações $\mathbf{x}=\mathbf{x}(t)$, ou

$$
\mathbf{x}=\left(\begin{array}{l}
x_{1}(t) \\
x_{2}(t) \\
x_{3}(t)
\end{array}\right),
$$

assim, cada ponto da trajetória está relacionado a um parâmetro $t$ através de um conjunto de equações paramétricas. Por exemplo, uma reta no eixo $\mathbf{e}_{1}$ é descrita pela equação $x=a+b t$, em que $a$ e $b$ são constantes. Os conceitos de curva e trajetória estão relacionados, mas não são iguais. A curva possui uma orientação definida, enquanto a trajetória não: uma partícula que vai de um ponto $A$ para um ponto $B$ sobre uma trajetória percorre o mesmo conjunto de pontos que uma partícula que vai de $B$ a $A$ pelo mesmo caminho. Assim, as trajetórias são iguais. Contudo, ambos os movimentos são descritos por curvas diferentes.

\section{O tempo}

O intervalo $I=\left[t_{0}, t\right]$ é um conjunto varrido por um parâmetro contínuo $t$ que, no geral, é arbitrário. A parametrização mais utilizada para uma curva é seu próprio comprimento, definido a partir da métrica euclidiana. Contudo, na mecânica classica existe uma parametrização especial, definida pelo seguinte postulado:

Postulado 4: O tempo é o parâmetro das curvas que determinam a trajetória das partículas e é definido pelo observador através de relógios. Possui as seguintes propriedades:

- O tempo é homogêneo e uniformemente crescente;

- Os intervalos de tempo são independentes dos observadores.

Assim, a mecânica clássica define um parâmetro "quase absoluto", definido por cada observador através de seu próprio relógio. Um relógio, neste caso, é um sistema físico oscilatório, que permite que o observador relacione um número real sempre que o relógio retorne a uma configuração de referência.

Ao postular que o tempo é homegêneo, estamos dando ao parâmetro $t$ a mesma liberdade que damos a um ponto do espaço euclidiano, o da escolha arbitrária da origem. Assim, o tempo inicial $t_{0}$ de uma curva é arbitrário e pode ser livremente escolhido por um observador. Portanto, dois observadores não precisam concordar com a origem do tempo, o que torna o tempo um conceito relativo: não existe tempo absoluto.

Contudo, os intervalos de tempo não são relativos. Por isso dizemos que a parametrização é quase absoluta. A segunda propriedade do postulado 4 nos diz que dois observadores precisam concordar com o intervalo de tempo sobre a curva. Portanto, $t-t_{0}$ para $t \geq t_{0}$ é invariante por translações na origem do tempo. Neste caso, o espaço do parâmetro temporal é um subespaço euclidiano unidimensional típico: é homogêneo e sua função distância é invariante por translações. O mesmo ocorre, se nos lembrarmos, com as coordenadas de distância em uma reta real.

O parâmetro temporal pertence, portanto, a um espaço homogêneo com métrica invariante. Contudo, há uma diferença com relação a uma coordenada de posição: na primeira propriedade, dissemos também que o tempo deve ser uniformemente crescente. O que isto significa? Se pensarmos bem na questão, isto só pode significar que uma curva tem comprimentos de trajetória iguais para tempos iguais. Do início da curva até seu final, o comprimento só pode crescer e, portanto, o tempo age da mesma forma. Pode apenas ser contado no sentido positivo de $\mathbb{R}$. Assim, o tempo deve ser proporcional ao comprimento da trajetória.

Há fortes razões empíricas para postularmos que a seta do tempo sempre aponte para frente. Deixarei que essas razões sejam descobertas pelo leitor, em uma tarefa de pesquisa que será definida no curso. 


\section{Suavidades das curvas}

Uma curva é contínua se a imagem recíproca de um conjunto aberto em $\mathbb{R}^{3}$ é um conjunto aberto em $I$. Intuitivamente, isto significa que pequenas variações no tempo implicam em pequenas variações no ponto da trajetória. Esta condição é fundamental para garantir que as trajetórias de partículas sejam contínuas no sentido intuitivo, ou seja, que ela não possa "aparecer" e "desaparecer" durante o movimento.

Contudo, continuidade não é suficiente. É necessário que as primeiras e segundas derivadas das curvas existam e sejam, também, contínuas. Isto é o que se definide como diferenciabilidade, ou suavidade. Se a primeira derivada da trajetória existe e é contínua, dizemos que a curva é de classe $C^{1}$. Se a segunda derivada existe e é contínua, a curva é de classe $C^{2}$. Uma curva suave, ou infinitamente diferenciável é o ideal para representar o movimento de uma partícula, ou seja, uma curva de classe $C^{\infty}$. Contudo, para a mecânica clássica, é suficiente que as curvas sejam de classe $C^{2}$. A razão será explicada mais adiante, em razão dos postulados dinâmicos.

\section{Velocidade}

A primeira derivada de uma curva é a velocidade. Assim, vamos tomar uma trajetória genérica $\mathbf{x}=\mathbf{x}(t)$, ou seja, descrita pelo conjunto de equações

$$
\begin{aligned}
& x_{1}=x_{1}(t), \\
& x_{2}=x_{2}(t), \\
& x_{3}=x_{3}(t) .
\end{aligned}
$$

Se a curva for ao menos de classe $C^{1}$, existem as primeiras derivadas

$$
\begin{aligned}
\frac{d x_{1}}{d t} & =\frac{d}{d t} x_{1}(t), \\
\frac{d x_{2}}{d t} & =\frac{d}{d t} x_{2}(t), \\
\frac{d x_{3}}{d t} & =\frac{d}{d t} x_{3}(t),
\end{aligned}
$$

que definem as velocidades nas direções $\mathbf{e}_{1}, \mathbf{e}_{2}$ e $\mathbf{e}_{3}$. Portanto, uma curva suave nos permite definir a velocidade de uma partícula sobre a trajetória:

$$
\mathbf{v} \equiv \frac{d \mathbf{x}}{d t} \equiv \dot{\mathbf{x}}
$$

em que o ponto acima do vetor posição indica derivada temporal. Assim, a velocidade é o vetor que é a derivada temporal da posição.

Como o tempo é monotonicamente crescente, isto significa que há uma relação linear do tempo com o comprimento da curva. Se denominarmos o comprimento da curva como $s$ e impusermos a condição inicial $t_{0}=0$ para $s=s_{0}=0$, temos que $s=u t$, em que $u$ é uma constante. Assim,

$$
\mathbf{v}=\frac{d \mathbf{x}}{d t}=\frac{d \mathbf{x}}{d s} \frac{d s}{d t}=u \frac{d \mathbf{x}}{d s} .
$$

Por outro lado, o vetor $d \mathbf{x} / d s$ é um vetor tangente à trajetória (lembrem-se de Cálculo C). Podemos definir um vetor tangente unitário através da relação

$$
\hat{T} \equiv \frac{d \mathbf{x} / d s}{|d \mathbf{x} / d s|},
$$

assim,

$$
\mathbf{v}=u\left|\frac{d \mathbf{x}}{d s}\right| \hat{T} \equiv v \hat{T},
$$

em que, claro, $v$ é o módulo da velocidade da partícula. Portanto, a velocidade é sempre tangente à trajetória. 


\section{Aceleração}

Caso a curva seja de classe $C^{2}$, a trajetória possui segunda derivada contínua. Isto nos permite introduzir a aceleração:

$$
\mathbf{a} \equiv \frac{d^{2} \mathbf{x}}{d t^{2}} \equiv \ddot{\mathbf{x}}
$$

que tem a seguinte relação com a velocidade:

$$
\mathbf{a}=\frac{d \mathbf{v}}{d t}=\dot{\mathbf{v}}
$$

Portanto, a aceleração é a derivada primeira da velocidade e a segunda derivada da posição.

Agora, tomemos a relação (??). A derivada desta relação implica em

$$
\dot{\mathbf{v}}=\dot{v} \hat{T}+v \frac{d \hat{T}}{d t}
$$

No primeiro termo, temos a aceleração tangencial da partícula:

$$
\mathbf{a}_{T} \equiv \dot{v} \hat{T},
$$

cuja norma é a derivada do módulo da velocidade. No segundo termo, temos a aceleração centrípeta

$$
\mathbf{a}_{C} \equiv v \frac{d \hat{T}}{d t},
$$

que pode ser escrita por

$$
\mathbf{a}_{C} \equiv v \frac{d \hat{T}}{d s} \frac{d s}{d t}=v u \frac{d \hat{T}}{d s} .
$$

Por outro lado, o vetor unitário definido por

$$
\hat{N} \equiv \frac{d \hat{T} / d s}{|d \hat{T} / d s|}
$$

é um vetor normal à trajetória $(\hat{T} \cdot \hat{N}=0)$. Assim,

$$
\mathbf{a}_{C}=v u\left|\frac{d \hat{T}}{d s}\right| \hat{N},
$$

portanto, o módulo da aceleração centrípeta depende da velocidade, da taxa de variação do comprimento da curva com o tempo e da norma da variação do vetor tangente com relação ao tempo. Esta norma é o que denominamos curvatura:

$$
\kappa \equiv\left|\frac{d \hat{T}}{d s}\right|,
$$

de modo que $\mathbf{a}_{C}=v u \kappa \hat{N}$.

Note, portanto, que a norma da aceleração não é a derivada da norma da velocidade, mas sim

$$
a \equiv|\mathbf{a}|=\sqrt{\dot{v}^{2}+(v u \kappa)^{2}}
$$

dependendo também da curvatura da trajetória. 


\section{Torção}

Se a trajetória da partícula é restrita ao plano formado por $\hat{T}$ e $\hat{N}$, e este plano não muda de orientação durante o movimento, a partícula fica restrita a este plano, portanto, podemos ver que este é o caso de um movimento bidimensional. Contudo, é possível que um movimento mais geral resulte que este plano, denominado plano osculante, mude com o tempo. Dizemos, assim, que a trajetória da partícula tem torção diferente de zero.

O vetor normal ao plano osculante com módulo positivo na direção da velocidade da partícula é denominado vetor binormal. Com o produto vetorial, podemos definir o vetor binormal através da relação

$$
\hat{B} \equiv \hat{T} \times \hat{N} .
$$

Abaixo, vamos introduzir o produto vetorial. Por enquanto, um plano osculante constante implica que o vetor binormal tem derivada nula com relação ao tempo, ou mais precisamente, com relação ao valor de comprimento da trajetória.

Se o vetor binormal não é constante na trajetória, temos

$$
\tau \equiv\left|\frac{d \hat{B}}{d s}\right|
$$

que define a torção da trajetória. Uma trajetória com torção não se restringe ao movimento da partícula em um único plano.

\section{O produto vetorial}

Em três dimensões, é possivel a definição de um produto antissimétrico que leva dois vetores a um vetor, o produto vetorial. Neste caso, o produto é definido por uma operação

$$
\bullet \times \bullet: \mathbb{R}^{3} \times \mathbb{R}^{3} \rightarrow \mathbb{R}^{3},
$$

com as seguintes propriedades ( $\operatorname{sejam} \mathbf{u}, \mathbf{v}$ e $\mathbf{w} \in \mathbb{R}^{3}$ e $\left.a, b \in \Phi\right)$ :

1. Antissimetria: $\mathbf{u} \times \mathbf{v}=-\mathbf{v} \times \mathbf{u}$;

2. Bilinearidade: $\mathbf{u} \times(a \mathbf{v}+b \mathbf{w})=a \mathbf{u} \times \mathbf{v}+b \mathbf{u} \times \mathbf{w}$;

3. Identidade de Jacobi: $\mathbf{u} \times(\mathbf{v} \times \mathbf{w})+\mathbf{v} \times(\mathbf{w} \times \mathbf{u})+\mathbf{w} \times(\mathbf{u} \times \mathbf{v})=0$.

Contudo, o produto vetorial não é associativo.

A expressão mais simples para o produto vetorial pode ser escrita pelo determinante

$$
\mathbf{u} \times \mathbf{v}=\operatorname{det}\left(\begin{array}{ccc}
\mathbf{e}_{1} & a m p ; \mathbf{e}_{2} & a m p ; \mathbf{e}_{3} \\
u_{1} & a m p ; u_{2} & a m p ; u_{3} \\
v_{1} & a m p ; v_{2} & a m p ; v_{2}
\end{array}\right)
$$

cuja norma é definida pela expressão

$$
|\mathbf{u} \times \mathbf{v}|=|\mathbf{u}||\mathbf{v}| \sin \theta
$$

em que $\theta$ é o ângulo entre os dois vetores. 